\title{
Relationship of Forest Vegetation to Soils on Geological Formations of the Oklahoma Gulf Coastal Plain
}

\author{
Raymond John Taylor, Professor Emeritus, deceased \\ Southeastern Oklahoma State University
}

\begin{abstract}
Dissertation submitted to the Graduate Faculty University of Oklahoma, Norman, Oklahoma, 1967
\end{abstract}

Portions of this paper were recently presented at the Cross Timbers Symposium at the Botany 2005 Symposium in Austin, Texas. I have come to realize the importance of this data as a baseline for the composition of forests in the Gulf Coastal Plain. Since the data were collected about 40 years ago, many changes have occurred including our expanding population, increase in rural housing, construction of pipelines, and clearing for pasture and cultivation. Many of the sites studied have been modified or completely disappeared. Another important change is the tremendous expansion of Juniperus virginiana (red cedar) due to absence of fire. In the sampling of these 13 forests 40 years ago, this species was found in only one stand, and as a sapling. Other invasive plants include Ligustrum sinense (privet), an evergreen shrub that can expand into clones by underground roots, and the invasive vine Lonicera sempervirens (Japanese honeysuckle) which is controlled in many areas by cattle grazing. Both of these species will continue to expand and affect our native flora.

Constance E. Taylor, Southeastern Oklahoma State University, Durant, Oklahoma, Professor Emeritus. Address: 621 S. Pirtle Rd., Durant, OK 74701

Email cetaylor@netcommander.com

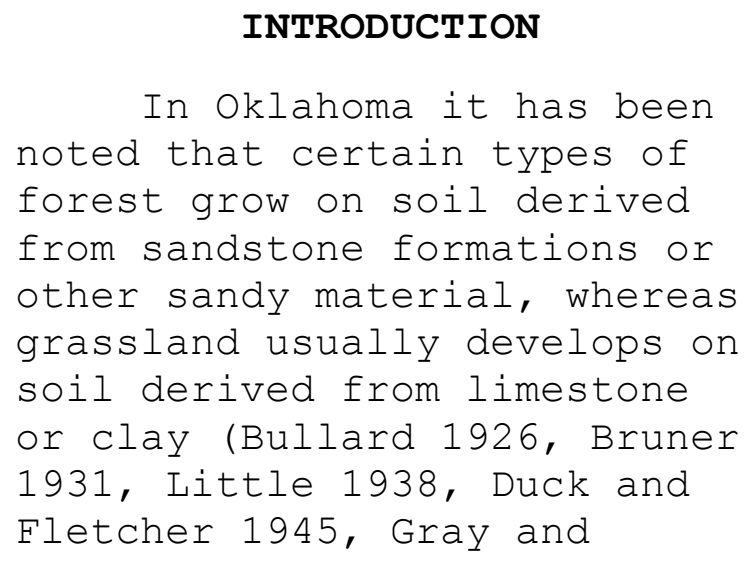

Taylor, R.J.

https://doi.org/10.22488/okstate.17.100037

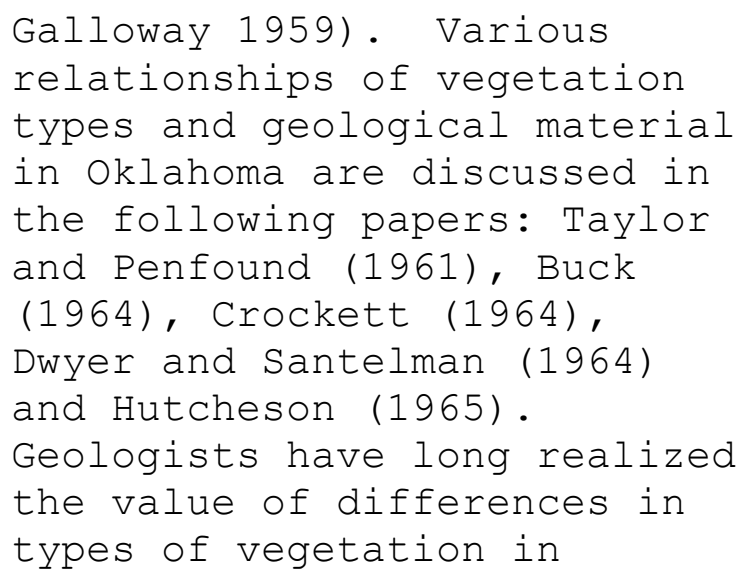


geological mapping (Cuyler 1931). The use of aerial photographs in geological mapping today is a standard practice (Gibbs 1950, Olson 1965 ).

Differences in

vegetation may often be related to differences in soil. Rice et al. (1960) found that three species of grass came into revegetating old fields in order of increasing nitrogen and phosphorus requirement. Beals and Cope (1964) found differences in herbaceous vegetation in Indiana forests associated with differences in drainage and soil moisture. Beadle(1966) discussed the role of soil phosphate in the molding of segments of the Australian flora. Mooney (1966) found that a number of soil properties were involved in the altitudinal distribution of two species of Erigeron. Porter (1966) found a difference in the distribution of two ecotypes of Panicum virgatum associated with a difference in nitrogen requirements. At the same time it is known that soil is influenced by the type of plants that grow on it. The role of certain legumes and a few other plants in increasing soil nitrogen is well documented. Eyer (1963) notes that quite different soil may develop under grasslands than under forest, even when the two areas lie side by side. Braun (1964) discussed the striking difference in color and texture in some islands of prairie soil that are surrounded by forest. Zinke (1962) found differences in soil under individual forest trees.

Thompson (1958) states

that a soil is the product of the interaction of parent material, climate, vegetation, topography, and time. Only a few studies that have dealt with the relationships between vegetation and geological material in Oklahoma have involved soil analysis. In this study the vegetation of 13 forest communities was analyzed. They are located in the Bryan county portion of the Oklahoma Gulf Coastal plain. The soil in which they grew was analyzed and the communities were correlated with soil and geological material.

The geological

formations of the old Cretaceous Gulf Coastal Plain is composed of sandstones, limestones, and clays that lie parallel, running east and west, but interrupted by a number of flood plains. Since much of the area is forested, it provides an excellent location for study of forest types and soils on different geological material.

Only communities growing on relatively level land and on soil derived from a particular recognizable geological formation were 
used. Ten upland and three bottomland communities were selected for investigation. These communities were located on Antlers sand (previously known as Paluxy sand of the Trinity Group (Forgotson 1957, and Redman, 1964), Pawpaw sand, Woodbine sand, Weno formation, Goodland limestone, Duck Creek limestone, Bennington limestone, Kiamichi clay, and alluvium from the flood plains of two rivers. All of these formations except the recent alluvium are Cretaceous in age.

The vegetation analysis included the following parameters: woody species grouped as 1) trees, 2) seedlings and saplings, 3) shrubs and vines; the mean area, frequency, density, size class, basal area, and plants per acre. The soil properties studied included the following: soil texture, $\mathrm{pH}$, organic carbon content, organic matter, nitrogen, phosphorus, volume-weight, and soil color.

The 13 communities enable comparison of 1 ) communities growing on soils derived from sandstone, limestone, clay, and alluvium; 2) two different stages of succession of alluvium soil (Red River communities); 3) alluvial soil from two stream systems; and 4) communities growing approximately 40 miles apart on the same formations (Pawpaw and Woodbine sands).

\section{DESCRIPTION OF AREA}

The Gulf Coastal plain of Oklahoma is located in the northwest portion of the Gulf Coastal Plain Province of Fenneman (1938). It is an area that extends from the Arkansas border westward to western Love County. The maximum north to south distance in Oklahoma is slightly over 35 miles. The main settlement of the area took place in 1832 when the tribes of the choctaw Indians were resettled in this part of Indian Territory.

Continuous cultivation dates from this time or slightly earlier.

The coastal plain is mainly forested, but with occasional strips of grassland mainly along the northern portion and becoming predominantly grassland in the western extent. Bruner (1931) classed the eastern half of the area as composed of an oak-hickory association, but oak-hickory savanna was the important type of vegetation in the western portion. Blair and Hubbell (1938) placed most of this area in their Osage Savanna grassland and allied it with the central part of the state. Duck and Fletcher (1945) listed approximately 3,600 square miles of forest and only 800 square miles of grassland for the Oklahoma Coastal Plain. Their forested areas contain five different types: loblolly pine, oak-pine, oak-hickory,

Taylor, R.J. 
post oak-blackjack oak, and bottomland. Braun (1964) refers to this area as a forest prairie transition. Rice and Penfound (1969) found post oak, blackjack oak, and black hickory to be the most important woody species in the coastal plain area. Kuchler (1964) maps the potential vegetation as oak-hickory-pine, oakhickory, cross timbers (post oak-blackjack oak), bluestem prairie, and the southern flood plain forest. The vegetation of this area has been subjected to fire and the influences of various kinds of agricultural practices since at least 1832 .

\section{CLIMATE}

The area in which the stands are located has a moist subhumid climate (Thornthwaite 1948). Average annual precipitation ranges from 36 inches in the southwest to slightly over 40 inches in the east (Wahlgren 1941). Average annual snowfall is 2.4 inches. Rainfall is relatively evenly distributed through the year with April, May, June, and July receiving heaviest amounts (Table I). The average annual temperature is $63.4^{\circ} \mathrm{F}$. with an average of $83^{\circ} \mathrm{F}$. in July and a low of $42^{\circ} \mathrm{F}$. in January. Frost free days are from about March 25 to Nov. 5, giving the county a 230 day growing season.
The Gulf Coastal plain in Oklahoma is characterized by low, eroded, gently rolling hills. Local relief is less than 100 feet (30.48 m) . There are also extensive areas of slightly undulating surface which are found mainly in the more northern portion. Elevation ranges from approximately 750 feet $(228.6 \mathrm{~m})$ in the north to slightly less than 450 feet $(137.16 \mathrm{~m})$ in the southwest. Resistant strata form northward facing escarpments with gentle dip slopes toward the south. One of these forms the ridge that crosses the county in an east-west direction near the middle of the study area. A second escarpment has been formed along the contact of the Goodland limestone and the Antlers sand. In general the study area slopes from north to south. Drainage is essentially dendritic, reflecting the relative uniform nature of the bedrock and lack of structural control. The area is drained principally by Island Bayou, Blue River, and White Grasses Creek into the Red River. The northeastern area is drained by Clear Boggy Creek. Several small streams along the west drain into the Washita arm of Lake Texoma. Extensive, relatively level flood plains are found along most of the rivers. Along some streams, natural levees have developed so that the portions of the flood plain farthest from the streams are

\section{TOPOGRAPHY}


lowest. As a result, these flood plains are inundated and wet during rainy periods for long periods. This has resulted in extensive swampy bottomland forests in many places. Minor topographical features of the area are bench like terraces which are found mainly along the Red River. There are also highlevel terrace remnants, presumably early Pleistocene in origin. The controversial pimple mounds (Melton, 1954) are found in many unplowed grasslands throughout the coastal plain with some in the immediate area of study stands. Another feature which none of the geologists who have worked here have discussed are the hillside seeps or bogs (Taylor and Taylor 1965).

\section{GEOLOGY}

Except for quaternary alluvium, only Cretaceous formations of the Comanchean and Gulfian series are recognized as occurring at the surface in the study area. At present no detailed geological study exists that covers all of it. The northern part was studied and mapped by Taff (1902, 1903). The region mapped by Stephenson (1919) covers the southeastern portion of the study area. The legend of the Geologic Map of Oklahoma (Miser 1954) contains much information about the geological studies done here. Hedlund (1962) studied the
Red branch member of the Woodbine formation. Recently a very fine study has been completed covering a large portion of Bryan county (Olson 1965). All but five of the stands and all geological formations of this study lie within his area of investigation.

Other investigations dealing with Cretaceous and Quaternary geology of the Gulf Coastal Plain of southeastern Oklahoma are listed: Love County (Bullard 1925); Marshall County (Bullard 1926); Choctaw County (Gibbs 1950); McCurtain County (Heilborn 1949, Skolnick 1949, and Davis 1960); Lower Cretaceous (Miser 1927); Trinity Group (Vanderpool 1928); Woodbine formation (Curtis 1960); and the Goodland Limestone (Blan 1961 ).

\section{STRATIGRAPHY, SOIL TYPES, AND STAND LOCATIONS}

The Cretaceous

formations occurring at the surface are considered to belong to two series, the Comanchean and Gulfian. The oldest, the Comanchean, has been further divided into three groups. Oldest to youngest, they are the Trinity, Fredericksburg, and Washita. A generalized columnar representation of the geological material found at the surface is shown in Figure 2. This columnar section is adapted mainly from Olson (1965). 
Soil types mapped for each stand were taken from the field data of Mr. Carter Steere, Soil Conservation Service, U.S. Department of Agriculture who was conducting a new soil survey of Bryan County.

Antlers Sand consists of approximately 300 feet (110 m) of loosely consolidated white to yellow cross-bedded pack sand inter-bedded with clay and sandy clay. At places there are moderately indurated layers of ironcemented sandstone up to 3 feet $(0.9 \mathrm{~m})$ thick. The soil formed from this material is generally a sandy or sandy loam with grayish brown surface and yellowish sand clay loam subsoil. Soil type is in the Bowie series. Usually forest vegetation develops on soil from this formation. Stand location is in Sec. 8, T5S-R13E, approximately 9.5 miles northeast of Bennington.

Goodland Limestone is a compact, finely crystalline limestone that becomes nodular near the bottom. Since it overlies the easily eroded Antlers Sand, a low escarpment forms along their contact. It is about 20 feet $(6.1 \mathrm{~m})$ thick in the study area. Soil formed from the Goodland is shallow clay to sandy clay loam, reddish black to dark reddish brown, only slightly differentiated in the lower portion. Soil type is in the Claremore series. Several types of vegetation develop on this formation from grassland to forest depending on occurrence of fire. The Goodland limestone stand was located in Sec. 7, T5S-R13E, approximately 9 miles northeast of Bennington.

Kiamichi Clay is mainly a black shaly clay which is thinly bedded with ironstained laminae. The upper portion is a hard yellowishbrown oyster shell limestone, large slabs of which break off as a result of slumping of the soft underlying clays, forming what is sometimes referred to as edge rock soils. This formation is about 35 feet $(10.67 \mathrm{~m})$ thick. Soils that develop from it consist of brown to dark brown clay at the surface with yellowish brown to brown clay subsoils. Soil Type is Denton edge rock series. The usual vegetation type on Kiamichi Clay is grassland. The forest stand studied was located in Sec. 8, T5S-R9E, approximately 6.5 miles northwest of Armstrong.

\section{Duck Creek Limestone}

consists of approximately 100 feet $(30.5 \mathrm{~m})$ of inter-bedded soft cream-colored limestone and bluish-gray shaly clay. The soil that develops on this formation is a very dark gray clay at the surface with a dark gray calcareous subsoil. The soil of the study stand was very shallow with limestone rock at the surface in many places. Soil type is in the San Saba series. Grassland is the usual vegetation on this 
soil, but in the absence of fire a weedy forest may develop. The Duck Creek stand is located approximately seven miles northwest of Armstrong in Sec. 5, T5S-R9E.

Weno Formation consists

of ferruginous sandstone, brownish marls, marly clays, and impure limestone. It is about 100 feet $(30.5 \mathrm{~m})$ thick in the study area. The soil has a yellowish-brown to grayish-brown sandy loam surface with a yellow red sandy clay loam subsurface. Its appearance at the surface was very similar to the Pawpaw sand which lies above it. Sometimes it is very difficult to distinguish between them. The vegetation is similar in appearance with no discernable break at their contact. However, this contact is usually marked by a thin limestone ledge, the quarry limestone, which occurs in the upper portion of the Weno. Soil type is in the Ruston series. Vegetation is forest unless cleared. The stand is located in Sec. 11, T5S-R7E, approximately four miles northwest of Mead.

Pawpaw Sand is composed mainly of yellow to red ferruginous sand inter-bedded with yellow to gray sandy clay and is about 50 feet $(15.24 \mathrm{~m})$ in depth. Soil formed was essentially like that of the Weno, being somewhat more sandy and more grayish brown at the surface. Soil type is in the Ruston series. Vegetation is forest unless cleared. The Pawpaw East stand is located in Sec. 12, T6S-R12E, approximately 4.5 miles northeast of Bennington. The Pawpaw West is located in Sec. 5, T6SR8E, approximately 2.5 miles northwest of Silo.

\section{Bennington Limestone} consists of 10 to 20 feet $(6.1 \mathrm{~m})$ of hard brownishyellow crystalline limestone. Olson (1965) listed a depth of seven feet $(2.1 \mathrm{~m})$ for eastern Bryan County. The soil of the forest stand studied was a shallow dark brown loam only slightly differentiated in the lower portion. Limestone rock occurred at the surface in many places. Soil type was in the Hunt series. An open, weedy, scrubby forest type of vegetation had developed in the absence of fire. The stand is located in Sec. 14, T6S-R12E, approximately two miles northeast of

Bennington.

Woodbine Formation is a series of red to tan soft moderately indurated ferruginous sands interbedded with silty clays and carbonaceous shale. It is over 300 feet $(91.4 \mathrm{~m})$ thick. The soil that developed in the study area was similar to that of the Pawpaw sand and is also in the Ruston series and supports forest vegetation. Two stands were studied: Woodbine East is located in Sec. 5, T7S-R13E, approximately five miles southeast of Bennington. The 
western stand is located in

Sec. 1, T7S-R7E, approximately 2 miles

southwest of Mead.

Red River Alluvium was

of two distinct types as indicated by the differences in soils and types of vegetation. The soils of the two Red River flood plain stands were similar, containing reddish brown clay in the surface soil with reddish sandy loam in the subsoil. In places, the subsoil had areas of clay several inches thick, and at others almost pure sand occurred. Soil types were in the Yahola series. Bottomland forests prevailed. An early succession forest stand, referred to as young Red River, was located in Sec. 22, T8S-R11E, approximately 3 miles southeast of Albany. The mature forest stand, referred to as old Red River, was located in Sec. 17, T8S-R14E, approximately 14.5 miles southeast of Bennington.

Clear Boggy alluvium had a very dark gray clay at the surface with a slightly lighter gray clay subsoil. The soil type is in the Osage series. The study stand is located in Sec. 8, T5S-R13E, 8.5 miles northeast of Bennington.

The location of the stands and the geology of the study area are shown in Fig. 1. This map is adapted mainly from Miser (1954) and Olsen (1965).

\section{METHODS}

After recognizance of the Gulf Coastal Plain strata exposed at the surface where forests occurred, 13 stands were selected for further study. To reduce effects of climate all stands were located in a 25 mile north to south by 40 mile east to west section of Bryan County, Oklahoma. Care was taken to select stands as mature as possible, and well within the area of outcrop of a particular formation. Only stands larger than 40 acres were utilized to permit a satisfactory sample.

The point-centered quarter method was used to obtain data for the vegetation analysis. This method was described and tested by cottam and Curtis (1956). They found that the types of distance methods commonly used in forest vegetation analysis, the quarter method gives least variable results for distance determinations, provides more data on tree species per sampling point, and is least susceptible to subjective bias. A series of points were established at predetermined intervals along a transect. A total of 25 points (100 quadrants) at 20 pace intervals were taken in each stand. The distance to the nearest individual in each of the four quadrants was determined. Data taken were species identification, d.b.h. (diameter at breast 
height). Data was also collected on shrubs and vines and seedlings and saplings.

Diameter at breast

height was used to compute basal area for each species and total basal area for the stand. Distance to the nearest individuals were averaged to obtain mean distance. The mean of all distances obtained from one stand has empirically (Cottam, Curtis, and Hale 1953) and theoretically (Morisita 1954) been shown to be equal to the square root of the mean area per plant. By dividing 43,560 square feet by the mean area per plant, the number of plants per acre was derived. The mean area and plants per acre for trees, seedlingssaplings, and shrubs-vines, was computed in this manner. The number of points at which a species was encountered divided by the total number of points times 100 was used to obtain species frequency within a stand. Density of a species was determined by taking the number of a species tallied for a stand divided by the number of quadrants times 100. Mean area, number of plants per acre, and mean distance are all related to density. The relative values for frequency, density, and basal area or dominance were computed by the formulas below.
Relative frequency $=$

frequency of a species x 100 total frequency of all species

Relative density $=$

density of a species x 100 total density of all species

Relative basal area =

basal area of a species $x 100$ total basal area of all species

Importance percentage of trees was obtained by adding relative frequency, relative density, and relative basal area and dividing by three. Importance percentage of seedling-saplings and shrubsvines was obtained by averaging relative frequency and relative density. Approximately 2,000 specimens of vascular plants have been collected from the study area, including at least one specimen of each species discussed. All were deposited at the Bebb Herbarium, University of Oklahoma, in Norman. Soil samples were taken from $0-6$ inches at 10 stations in each area. Only the 0-6 inch layer was sampled as soil covering most of the limestones was so shallow it was difficult to sample even to this depth in many places. Stations were evenly distributed in stand. A soil auger was used, with care taken to remove all duff

Taylor, R.J. 
Total nitrogen was determined by the method of Noggle and Wynd (1941). All determinations were run in duplicate. If the values for the two samples were essentially the same, they were averaged and this value used. If the two samples varied more than a few points, additional samples were analyzed. Volume-weight (soil compaction) was determined by the following method. Ten holes per stand approximately 2 inches in diameter and 3 inches deep were excavated. Soil from each hole was collected and later oven dried. Then each hole was filled with oven-dry quartz sand and the volume recorded. The oven-dry weight of the collected soil was determined and compaction was calculated by dividing this weight in grams by the cubic centimeters of sand. The air dry color of each soil sample was determined with the aid of a Munsell color chart. 


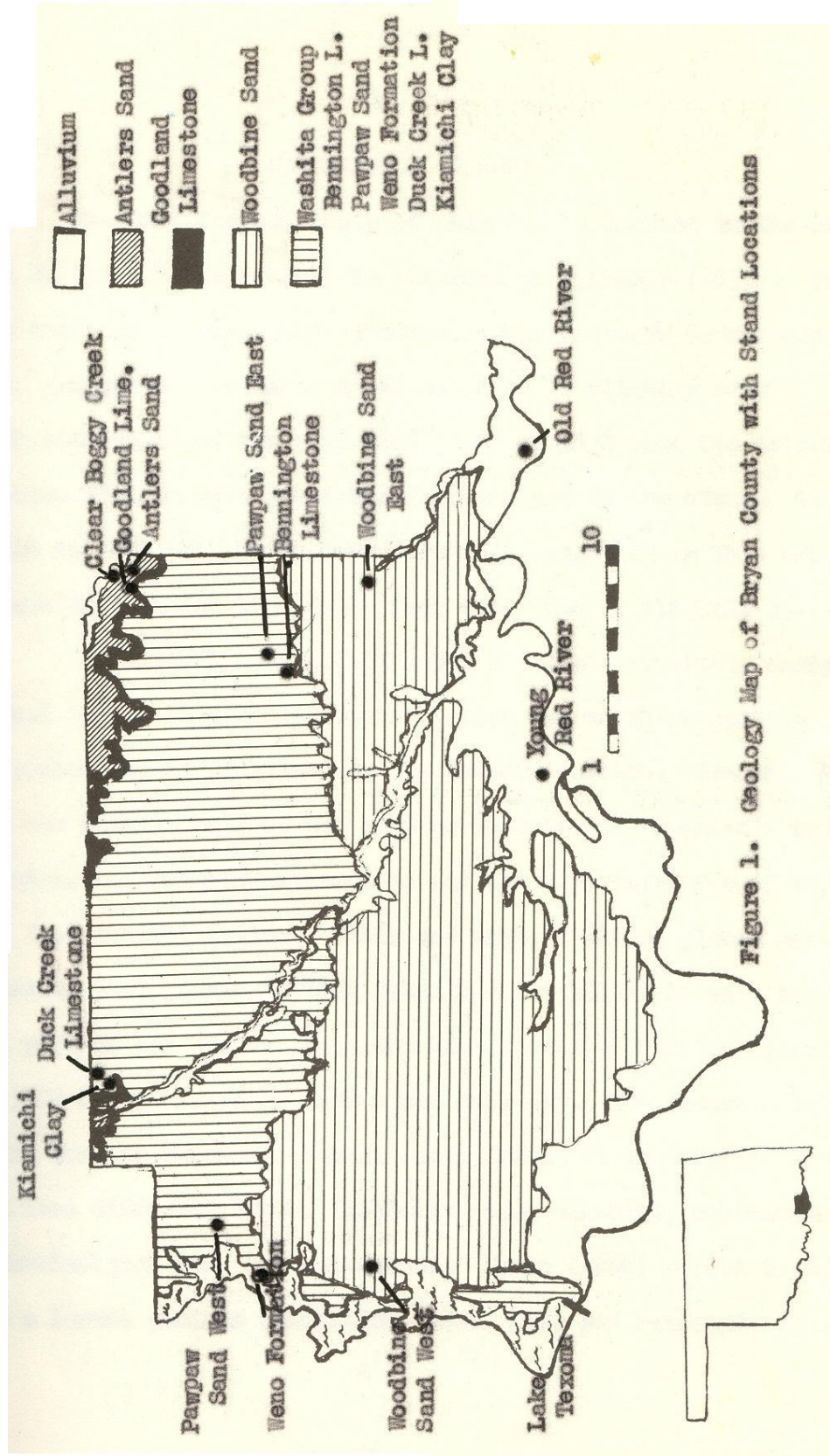

Taylor, R.J. 


\begin{tabular}{|c|c|c|c|}
\hline Age & Series & Group & Formation \\
\hline Recent & & & Mainly Alluvium \\
\hline \multirow[t]{2}{*}{ Pleistocene } & & & Terrace and Alluvium \\
\hline & Gulfian & & $\begin{array}{l}\text { Eagle Ford Shale } \\
\text { Woodbine Formation }\end{array}$ \\
\hline \multirow[t]{3}{*}{ Cretaceous } & \multirow[t]{3}{*}{ Comanchean } & Washita & $\begin{array}{l}\text { Bennington Limestone } \\
\text { Pawpaw Sand } \\
\text { Weno Formation } \\
\text { Denton Clay } \\
\text { Fort Worth Limestone } \\
\text { Duck Creek Limestone }\end{array}$ \\
\hline & & Fredericksburg & $\begin{array}{l}\text { Kiamichi Clay } \\
\text { Goodland Limestone }\end{array}$ \\
\hline & & Trinity & Antlers Sand \\
\hline
\end{tabular}

Figure 2 A generalized columnar representation of the geological material found at the surface in the study area 
Table I Mean precipitation and temperature based on 57-60 years of weather data

\begin{tabular}{lcc}
\hline Month & $\begin{array}{c}\text { Average Monthly } \\
\text { Precipitation in Inches }\end{array}$ & $\begin{array}{c}\text { Average Monthly } \\
\text { Temperature in Degrees F }\end{array}$ \\
\hline January & 2.24 & 42.4 \\
February & 2.59 & 45.9 \\
March & 2.87 & 54.6 \\
April & 4.52 & 63.0 \\
May & 5.40 & 70.5 \\
June & 3.75 & 79.0 \\
July & 3.21 & 83.0 \\
August & 2.75 & 83.2 \\
September & 3.03 & 76.3 \\
October & 3.77 & 65.2 \\
November & 2.62 & 53.2 \\
December & 2.62 & 44.3 \\
Yearly Average & 39.37 & 63.4 \\
\hline Data from U. S. Department of Commerce, Weather Bureau, Climatography of the United States
\end{tabular}

Taylor, R.J. 
Table II Importance percentage of trees in the 13 forest stands studied. An x represents a value of less than 5 per cent.

\begin{tabular}{|c|c|c|c|c|c|c|c|c|c|c|c|c|c|}
\hline Species & BB & OR & YR & A I & NE & WW & PE I & PW & WF & GL & $\mathrm{BL}$ & $\mathrm{DC}$ & $\mathrm{KC}$ \\
\hline $\begin{array}{l}\text { Ulmus crassifolia } \\
\text { Celtis laevigata } \\
\text { Fraxinus pennsylvanica } \\
\text { Maclura pomifera } \\
\text { Quercus phellos }\end{array}$ & $\begin{array}{r}26 \\
27 \\
25 \\
8 \\
9\end{array}$ & $\begin{array}{l}35 \\
19\end{array}$ & $\begin{array}{c}11 \\
\mathrm{X}\end{array}$ & $\begin{array}{l}X \\
X\end{array}$ & & & & & & $\begin{array}{l}X \\
X\end{array}$ & $\begin{array}{l}34 \\
27\end{array}$ & $\begin{array}{c}67 \\
x \\
25\end{array}$ & $\begin{array}{l}75 \\
25\end{array}$ \\
\hline $\begin{array}{l}\text { Q. nigra } \\
\text { Q. macrocarpa } \\
\text { Ulmus rubra } \\
\text { Crataegus spp. } \\
\text { Carya illinoensis }\end{array}$ & $\begin{array}{l}\mathrm{X} \\
\mathrm{X} \\
\mathrm{X} \\
\mathrm{X}\end{array}$ & 35 & $\mathrm{X}$ & & & & $\mathrm{x}$ & & & & $\mathrm{x}$ & & \\
\hline $\begin{array}{l}\text { Acer negundo } \\
\text { Diospyros virginiana } \\
\text { Gleditsia triacanthos } \\
\text { Morus rubra } \\
\text { Platanus occidentalis }\end{array}$ & & $\begin{array}{l}\mathrm{X} \\
\mathrm{X} \\
\mathrm{X} \\
\mathrm{X} \\
\mathrm{X}\end{array}$ & $\begin{array}{l}\mathrm{X} \\
\mathrm{x}\end{array}$ & $\mathrm{x}$ & $\begin{array}{l}x \\
X\end{array}$ & $\mathrm{x}$ & & & & $\mathrm{x}$ & 11 & $\mathrm{x}$ & \\
\hline $\begin{array}{l}\text { Populus deltoides } \\
\text { Salix nigra } \\
\text { Carya texana } \\
\text { Quercus stellata } \\
\text { Q. velutina } \\
\end{array}$ & & & $\begin{array}{r}74 \\
7\end{array}$ & $\begin{array}{l}37 \\
27 \\
13\end{array}$ & $\begin{array}{c}9 \\
38 \\
13 \\
\end{array}$ & $\begin{array}{c}16 \\
45 \\
21 \\
\end{array}$ & $\begin{array}{l}42 \\
17 \\
13\end{array}$ & $\begin{array}{l}31 \\
30 \\
11 \\
\end{array}$ & $\begin{array}{c}\mathrm{X} \\
68\end{array}$ & $\begin{array}{r}29 \\
20 \\
\times \\
\end{array}$ & & & \\
\hline $\begin{array}{l}\text { Q. falcata } \\
\text { Q. marilandica } \\
\text { Ulmus alata } \\
\text { Cercis canadensis } \\
\text { Ulmus americana }\end{array}$ & & & & $\begin{array}{l}\mathrm{X} \\
\mathrm{X} \\
\mathrm{X} \\
\mathrm{X} \\
\mathrm{X}\end{array}$ & $\begin{array}{r}17 \\
\mathrm{x} \\
10\end{array}$ & $\begin{array}{r}12 \\
\mathrm{X} \\
\mathrm{X}\end{array}$ & $\begin{array}{r}\mathrm{X} \\
10 \\
5\end{array}$ & $\begin{array}{r}19 \\
7 \\
\mathrm{X} \\
\mathrm{X}\end{array}$ & 13 & $\begin{array}{l}9 \\
\mathrm{x} \\
\mathrm{x} \\
\mathrm{x}\end{array}$ & $\begin{array}{r}16 \\
x\end{array}$ & & \\
\hline $\begin{array}{l}\text { Carya tomentosa } \\
\text { Fraxinus americana } \\
\text { Quercus shumardii } \\
\text { Bumelia lanuginosa } \\
\text { Q. muehlenbergii } \\
\text { Prunus spp. } \\
\text { Q. rubra }\end{array}$ & & & & $\mathrm{x}$ & $\mathrm{x}$ & & 8 & & $\begin{array}{c}17 \\
\mathrm{X}\end{array}$ & $\begin{array}{l}\mathrm{X} \\
5 \\
8 \\
\mathrm{X} \\
\mathrm{X} \\
\mathrm{X}\end{array}$ & $\begin{array}{l}X \\
X \\
X\end{array}$ & $\mathrm{X}$ & \\
\hline
\end{tabular}

BB - Boggy Creek Bottomland

OR - Old Red River Alluvium

YR - Young Red River Alluvium

A - Antlers sand

WE - Woodbine East
WW - Woodbine West

PE - Pawpaw East

PW - Pawpaw West

WE - Weno Formation

GL - Goodland Limestone
BL - Bennington Limestone

DC - Duck Creek Limestone

KC - Kiamichi Clay

Taylor, R.J. 
Table III Number of woody plants, basal area, and d.b.h. or trees in the forest stands investigated

\begin{tabular}{|c|c|c|c|c|c|}
\hline Stands & $\begin{array}{l}\text { Trees } \\
\text { per acre }\end{array}$ & $\begin{array}{l}\text { Mean } \\
\text { d.b.h. in } \\
\text { Inches }\end{array}$ & $\begin{array}{c}\text { BA/Acre } \\
\text { in } \\
\text { sq. feet }\end{array}$ & $\begin{array}{l}\text { Seedlings } \\
\text { Saplings } \\
\text { per acre }\end{array}$ & $\begin{array}{l}\text { Shrubs } \\
\text { Vines } \\
\text { per acre }\end{array}$ \\
\hline \multicolumn{6}{|l|}{ Bottomland Stands } \\
\hline $\begin{array}{l}\text { Clear Boggy Creek } \\
\text { Red River Alluvium }\end{array}$ & 111.2 & 11.91 & 104.2 & 2,807 & 105 \\
\hline Young Stand & 112.6 & 8.45 & 51.7 & 144 & 3,457 \\
\hline Old Stand & 190.0 & 10.53 & 142.5 & 358 & 3,723 \\
\hline \multicolumn{6}{|l|}{ Upland Stands } \\
\hline \multicolumn{6}{|l|}{ Sandstone Formations } \\
\hline Antlers Sand & 244.8 & 7.32 & 88.7 & 6,396 & 7,169 \\
\hline Woodbine East & 171.0 & 8.90 & 90.3 & 6,443 & 5,556 \\
\hline Woodbine West & 254.6 & 6.69 & 75.3 & 1,308 & 8,377 \\
\hline Pawpaw East & 240.4 & 6.13 & 65.7 & 4,229 & 3,556 \\
\hline Pawpaw West & 160.4 & 8.40 & 81.9 & 8,677 & 3,704 \\
\hline Weno Formation & 245.1 & 6.10 & 61.6 & 2.774 & 3,723 \\
\hline \multicolumn{6}{|l|}{ Limestones and Clays } \\
\hline Goodland Limestone & 277.5 & 6.76 & 85.1 & 9,248 & 2,074 \\
\hline Bennington L. & 235.2 & 6.82 & 86.8 & 1,571 & 259 \\
\hline Duck Creek L. & 173.0 & 5.09 & 26.0 & 486 & - \\
\hline Kiamichi Clay & 265.4 & 5.68 & 62.4 & 563 & 511 \\
\hline Stands Average & 206.3 & 7.59 & 78.6 & $3,461.8$ & $3,247.2$ \\
\hline
\end{tabular}


Table IV Importance percentage of seedlings and saplings in the 13 forest stands studied. An $\mathrm{x}$ represents a value of less than 5 per cent.

\begin{tabular}{|c|c|c|c|c|c|c|c|c|c|c|c|c|c|}
\hline Species & $\mathrm{BB}$ & OR & YR & A & $\mathrm{NE}$ & WW & $\mathrm{PE}$ & PW & WF & GL & BL & $\mathrm{DC}$ & $\mathrm{KC}$ \\
\hline $\begin{array}{l}\text { Fraxinus pennsylvanica } \\
\text { Celtis laevigata } \\
\text { Ulmus crassifolia } \\
\text { Gleditsia triacanthos } \\
\text { Sapindus drummondii }\end{array}$ & $\begin{array}{c}56 \\
37 \\
x \\
x\end{array}$ & $\begin{array}{r}26 \\
\\
x \\
26\end{array}$ & $\begin{array}{c}22 \\
x\end{array}$ & $\begin{array}{l}15 \\
10\end{array}$ & $\mathrm{x}$ & & & $\mathrm{x}$ & $\begin{array}{l}\mathrm{x} \\
\mathrm{x}\end{array}$ & $\begin{array}{r}14 \\
\mathrm{x}\end{array}$ & $\begin{array}{l}12 \\
22 \\
16\end{array}$ & $\begin{array}{r}35 \\
45 \\
x\end{array}$ & $\begin{array}{r}15 \\
18 \\
37 \\
6\end{array}$ \\
\hline $\begin{array}{l}\text { Morus rubra } \\
\text { Ulmus rubra } \\
\text { Maclura pomifera } \\
\text { Acer negundo. } \\
\text { Carya illinoensis }\end{array}$ & & $\begin{array}{r}15 \\
13 \\
6 \\
\mathrm{X} \\
\mathrm{x}\end{array}$ & $\begin{array}{l}10 \\
\mathrm{X} \\
\mathrm{X} \\
\mathrm{X}\end{array}$ & 8 & & $\mathrm{x}$ & & & & $\mathrm{x}$ & $\begin{array}{l}x \\
15\end{array}$ & 14 & 10 \\
\hline $\begin{array}{l}\text { Sophora affinis } \\
\text { Quercus muehlenbergii } \\
\text { Ulmus americana } \\
\text { Salix nigra } \\
\text { Populus deltoides }\end{array}$ & & $\begin{array}{l}\mathrm{x} \\
\mathrm{x} \\
\mathrm{X}\end{array}$ & $\begin{array}{c}x \\
20 \\
16\end{array}$ & & & & & & & $\begin{array}{l}x \\
x\end{array}$ & & & $\mathrm{x}$ \\
\hline $\begin{array}{l}\text { Juniperus virginiana } \\
\text { Diospyros virginiana } \\
\text { Cornus florida } \\
\text { Quercus velutina } \\
\text { Ulmus alata }\end{array}$ & & & $\begin{array}{r}11 \\
\mathrm{X} \\
\mathrm{X} \\
\mathrm{X}\end{array}$ & $\begin{array}{r}5 \\
48\end{array}$ & $\begin{array}{c}x \\
6 \\
7 \\
58\end{array}$ & $\begin{array}{l}17 \\
52\end{array}$ & $\begin{array}{l}16 \\
48\end{array}$ & $\begin{array}{c}x \\
12 \\
37\end{array}$ & 39 & $\begin{array}{r}x \\
x \\
35\end{array}$ & $\begin{array}{l}5 \\
x \\
17\end{array}$ & & \\
\hline $\begin{array}{l}\text { Quercus stellata } \\
\text { Carya texana } \\
\text { Q. marilandica } \\
\text { Q. rubra } \\
\text { Q. falcata }\end{array}$ & & & & $\begin{array}{r}18 \\
7 \\
\mathrm{x} \\
\mathrm{x} \\
\mathrm{x}\end{array}$ & $\begin{array}{r}x \\
10\end{array}$ & $\begin{array}{r}22 \\
\mathrm{X} \\
\mathrm{X}\end{array}$ & $\begin{array}{r}17 \\
8 \\
\mathrm{X} \\
\mathrm{X}\end{array}$ & $\begin{array}{r}12 \\
17 \\
X\end{array}$ & $\begin{array}{r}25 \\
\mathrm{x}\end{array}$ & $\begin{array}{l}5 \\
5\end{array}$ & & & \\
\hline $\begin{array}{l}\text { Sassafras albidum } \\
\text { Prunus spp. } \\
\text { Carya tomentosa } \\
\text { Cercis canadensis } \\
\text { Fraxinus americana }\end{array}$ & & & & $\begin{array}{l}x \\
X\end{array}$ & $\mathrm{X}$ & & $\mathrm{X}$ & $\begin{array}{r}9 \\
19\end{array}$ & $\mathrm{X}$ & $\begin{array}{r}6 \\
19\end{array}$ & & & \\
\hline $\begin{array}{l}\text { Bumelia lanuginosa } \\
\text { Quercus shumardii } \\
\text { Crataegus spp. } \\
\text { Q. nigra }\end{array}$ & & & & & & & & $\mathrm{x}$ & $\mathrm{x}$ & $\mathrm{x}$ & $\begin{array}{l}\mathrm{X} \\
\mathrm{X}\end{array}$ & $\mathrm{X}$ & $\mathrm{X}$ \\
\hline
\end{tabular}

BB - Boggy Creek Bottomland

OR - Old Red River Alluvium

YR - Young Red River Alluvium

A - Antlers sand

WE - Woodbine East
WW - Woodbine West

PE - Pawpaw East

PW - Pawpaw West

WE - Weno Formation

GL - Goodland Limestone
BL - Bennington Limestone

DC - Duck Creek Limestone

KC - Kiamichi Clay

Taylor, R.J. 
Table V Importance percentage of common shrubs and vines in the13 forest stands studied. An $\mathrm{x}$ represents a value of less than 5 per cent.

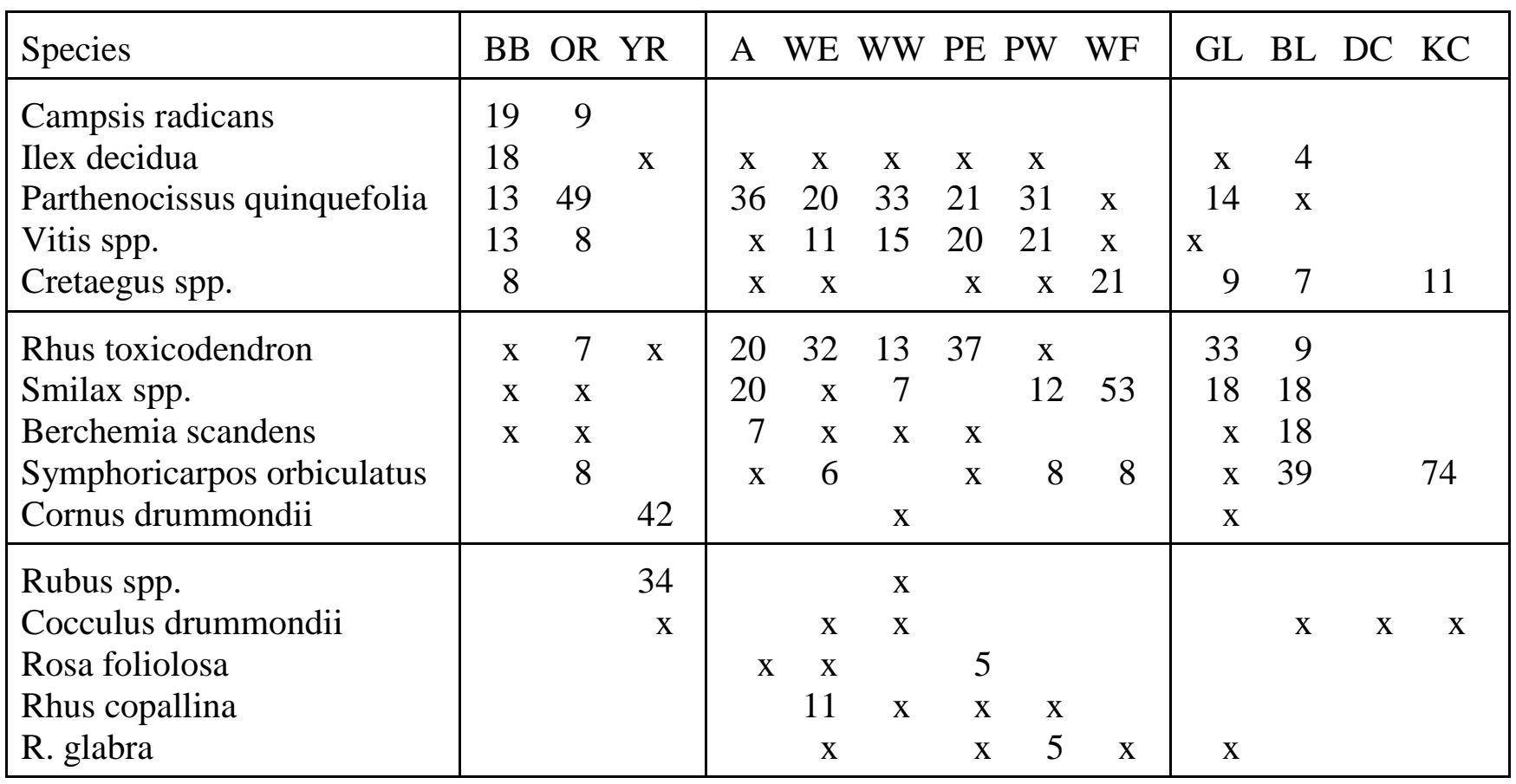

BB - Boggy Creek Bottomland

OR - Old Red River Alluvium

YR - Young Red River Alluvium

A - Antlers sand

WE - Woodbine East
WW - Woodbine West

PE - Pawpaw East

PW - Pawpaw West

WE - Weno Formation
BL - Bennington Limestone

DC - Duck Creek Limestone

KC - Kiamichi Clay

GL - Goodland Limestone 
Table VI Physical and chemical soil factors of the study stands. Pounds per acre are based on an acre furrow slice.

\begin{tabular}{|c|c|c|c|c|c|c|c|c|c|}
\hline Stands & $\begin{array}{l}\% \\
\text { Sand }\end{array}$ & $\begin{array}{c}\% \\
\text { Silt }\end{array}$ & $\begin{array}{l}\% \\
\text { Clay }\end{array}$ & $\mathrm{pH}$ & $\begin{array}{l}\text { Total } \\
\text { Phosphorus } \\
\text { Lbs./acre }\end{array}$ & $\begin{array}{l}\text { Total } \\
\text { Nitrogen } \\
\text { Lbs/acre }\end{array}$ & $\begin{array}{l}\text { Total } \\
\text { Carbon } \\
\text { Lbs/acre }\end{array}$ & $\frac{\mathrm{C}}{\mathrm{N}}$ & $\begin{array}{l}\text { Compaction } \\
\text { g/cc }\end{array}$ \\
\hline \multicolumn{10}{|l|}{ Bottomland Stands } \\
\hline $\begin{array}{l}\text { Clear Boggy Creek } \\
\text { Red River Alluvium }\end{array}$ & 20.1 & 12.9 & 67.0 & 8.0 & 2,273 & 3,666 & 34,053 & 9.29 & 1.05 \\
\hline Young Stand & 28.6 & 19.4 & 52.0 & 7.6 & 1,664 & 1,594 & 12,688 & 7.96 & 0.92 \\
\hline Old Stand & 27.2 & 21.9 & 50.9 & 7.4 & 2,088 & 2,488 & 19,458 & 7.82 & 0.96 \\
\hline \multicolumn{10}{|l|}{ Upland Stands } \\
\hline \multicolumn{10}{|l|}{ Sandstone Formations } \\
\hline Antlers Sand & 86.6 & 7.0 & 6.4 & 7.3 & 301 & 442 & 4,925 & 11.14 & 1.13 \\
\hline Woodbine East & 77.8 & 15.2 & 7.0 & 7.2 & 203 & 260 & 4,022 & 15.48 & 1.08 \\
\hline Woodbine West & 88.2 & 7.3 & 4.5 & 6.9 & 287 & 350 & 5,060 & 14.46 & 0.98 \\
\hline Pawpaw East & 85.6 & 8.4 & 6.0 & 6.2 & 309 & 556 & 5,340 & 9.60 & 1.03 \\
\hline Pawpaw West & 88.9 & 5.4 & 5.7 & 6.6 & 541 & 314 & 6,470 & 20.61 & 1.03 \\
\hline Weno Formation & 23.6 & 55.6 & 20.8 & 6.2 & 1,154 & 3,615 & 9,822 & 7.10 & 1.38 \\
\hline \multicolumn{10}{|l|}{ Limestone Formations } \\
\hline Goodland Limestone & 62.6 & 13.4 & 24.0 & 7.8 & 1,956 & 5,450 & 52,716 & 9.67 & 1.17 \\
\hline Bennington Limestone & 43.6 & 45.9 & 10.5 & 7.3 & 1,861 & 2,768 & 33,139 & 11.97 & 1.27 \\
\hline Duck Creek Limestone & 35.6 & 21.1 & 43.3 & 7.6 & 1,868 & 4,332 & 40,471 & 9.34 & 1.01 \\
\hline Kiamichi Clay & 35.6 & 14.4 & 50.0 & 7.2 & 2,149 & 6,074 & 55,115 & 9.08 & 1.2 \\
\hline
\end{tabular}




\section{RESULTS}

Vegetation and Soil Relationships in Individual Stands

\section{Clear Boggy Creek Stand}

Vegetation in this stand was an open elm-ash-hackberry community. It was not typical of this type in Oklahoma as the species of elm was neither Ulmus americana (American elm)nor U. rubra (slippery elm), but U. crassifolia (cedar elm) (Table II). It was the most open stand but had the next to highest basal area of 104 sq. ft. per acre. The average d.b.h of the stand was 11.9 inches (Table III). This type of community occurred in a number of places elsewhere in the Oklahoma Gulf coastal plain, especially in the western part, but it does not seem to have been previously described for the state. This is cover type 85 of the Society of American Foresters (1931). All further cover types mentioned are from this source. Seedlings and saplings indicate that Fraxinus pennsylvanica (green ash) and Celtis laevigata (hackberry) were becoming increasingly important, whereas there were only a few seedlings of $U$. crassifolia (Table IV). Ilex decidua (deciduous holly) was the most common shrub, while Campsis radicans (trumpet vine), Parthenocissus quinquefolia (Virginia creeper) and Vitis spp. (wild grape) were the more common vines (Table V).

The soil of this stand was a dark gray, moderately alkaline clay. The $\mathrm{pH}$ of 8 was the highest soil reaction for any stand, and was rather high in phosphorus, nitrogen, and organic carbon (Table VI). Since it was often inundated for extended periods each year, there were several layers of dark gray black sediment.

\section{Young Red River Stand}

vegetation was primarily of Populus deltoides (cottonwood) and corresponds to forest type 61 (Table II). It usually succeeds Salix nigra (black willow) and $S$. interior (now $S$. exigua, sandbar or coyote willow), and is followed by green ash, species of elm, and

hackberry. of all stands it had the next to lowest basal area of $54 \mathrm{sq}$. ft. per acre and was one of the most open stands (Table III). The mean d.b.h. for this stand is 8.5 inches. The number of seedlings and saplings indicated that Fraxinus pennsylvanica (green ash) had already become an important member although black willow still persisted.

Reproduction was largely by black willow, but a number of green ash, red mulberry, eastern red cedar, and an occasional American elm forecast a change to an elmash-hackberry community (Table IV). Cornus 
drummondii (rough-leaved Dogwood) formed an important part of the understory with Rubus spp. (blackberry) and Rhus toxicodendron (poison ivy) being the main vines (Table V) . The soil was a light reddish brown, slightly alkaline clay, and was fertile compared with soils of other stands (Table VI). It was interesting to note how closely it resembled the soil of the more mature Red River floodplain stand. Sand is occasionally encountered from a few inches down to a foot or more. At other locations, clay may be found down to the three foot level. Ditches cut by farmers for drainage or other purposes showed almost pure sand was encountered at varying distances below a layer of reddish clay. Where the river moved south in its meanderings, dune areas usually formed north of the sandy bed recently vacated. Before the impoundment of Lake Texoma, these dune areas were periodically inundated by the muddy water of the Red River. Sediment coming mainly from the Permian Red beds farther west was deposited over the dunes, forming the present surface. The soil of the flood plain is one of the most fertile and most productive in southeastern Oklahoma. The effect of the stage of succession or maturity of a forest community on the soil in which it grows was well demonstrated by the difference of total phosphate, total nitrogen, and organic matter (Table VI) between this stand and the Old Red River Stand.

Old Red River stand was a relatively typical elm-ashhackberry bottomland forest and corresponded to forest cover type 85. Ulmus rubra (slippery elm) was the main species of elm. The forest basal area of $143 \mathrm{sq}$. ft. per acre was highest of any stand. It had the next highest d.b.h. which averaged 10.5 inches but the stand was relatively open (Table III). The Society of Foresters (1931) considered this type temporary and one which developed after heavy cutting. However, the study stand showed no sign of ever having been lumbered. In the central and west central part of Oklahoma, elm-ashhackberry is the usual type of most mature bottomland stands (Bruner 1931). Associated species such as Quercus macrocarpa (bur oak), Q. shumardii (Shumard's red oak), and $Q$. muehlenbergii (chinquapin or chestnut oak) seldom become dominant and usually compose a minor portion of such stands. Further west, near the western border of Oklahoma and in the Panhandle, willow and cottonwood persist without being succeeded by elm-ash-hackberry. Perhaps as one moves away from optimum the stages to which 
succession can proceed becomes successively lower. Thus willow and cottonwood persist in the Panhandle, elm-ash-hackberry occurs in the central and western twothirds of the state, and oaks, gums, maples, and cypress are common in the east. The seedlings of Ulmus rubra (slippery elm) and Celtis laevigata (hackberry) made up a large portion of the reproduction of the stand, with Fraxinus pennsylvanica (green ash) comprising only a minor portion (Table IV). The most common shrub was Symphoricarpos orbiculata (coral berry, buckbrush) with campsis radicans (trumpet vine), Parthenocissus quinquefolia (Virginia creeper), and Vitis spp. (wild grape) as the more common vines (Table V).

The physical properties of the soil of this stand were very close to that of the New Red River stand. It has approximately a forth more total phosphorus and about a third more total nitrogen and carbon per acre (Table VI) .

\section{Antlers Sand Stand} supported a good upland forest principally of Quercus stellata (post oak) and Carya texana (black or pignut hickory) (Table II). The basal area of $89 \mathrm{sq}$. ft. per acre. for this stand was next to the highest for the sandy soils. It was a relatively closed stand with an average d.b.h. of 7.3 inches (Table III). Ulmus alata (winged elm) had the highest number of seedlings and saplings. There were also seedlings of Quercus stellata, Celtis laevigata (hackberry), $Q$. velutina (black oak), and Fraxinus pennsylvanica (green ash) (Table IV). Only an occasional shrub was encountered, but Smilax spp. (greenbriar), Rhus toxicodendron (poison ivy), and Parthenocissus quinquefolia (Virginia creeper) were common vines in the understory (Table V). The surface soil is a light yellowish brown, essentially neutral sand. It is low in fertility and apparently very susceptible to leaching (Table VI).

\section{Woodbine Sand Stands}

were dominated by Quercus stellata (post oak), $Q$. velutina (black oak), and Carya texana (black or pignut hickory). Quercus falcata (spanish oak) and Carya tomentosa (now called $C$. alba, mockernut hickory), occur in the eastern stand with the former having a slightly higher importance percentage than $Q$. velutina; neither were found in the western stand (Table II). The basal area of $90 \mathrm{sq}$. ft. per acre was highest of any sandstone stand (Table III). Ulmus alata (winged elm) had the highest number of seedlings and saplings in 
both stands as it did in the other sandstone stands and the Goodland limestone and Weno formation stands. However, it formed a minor portion of the tree canopy (Tables III and IV). Shrubs were not common in the understory, but three vines, Rhus toxicodendron (poison ivy), Parthenocissus quinquefolia (Virginia creeper), and Vitis spp (wild grape) were relatively common (Table V) .

The surface soil of these two stands, with some slight exceptions, were very much alike, although they were located 40 miles apart. Their color, texture, $\mathrm{pH}$, and phosphorus content were essentially the same (Table VI). The slightly lower nitrogen, carbon, and organic matter content, as well as the slightly lower nitrogen, carbon, and organic matter content, as well as the slightly higher volume weight of the soil of the eastern stand might have been the result of slightly increased leaching. The eastern stand was somewhat more open and occurred in an area with about four inches more annual precipitation. These facts might have contributed to increased leaching in the eastern stand.

Pawpaw Sand Stands had vegetation that was essentially the same with Carya texana (black or pignut hickory), Quercus stellata (post oak), and Q. velutina (black oak) having higher importance percentages in the eastern stand. Quercus marilandica (blackjack oak) replaced $Q$. velutina in importance in the western stand. Quercus falcata (spanish oak) and Carya tomentosa (now C. alba, mockernut hickory) were absent from the latter (Table II). The eastern stand had the lowest basal area, $66 \mathrm{sq}$. ft. per acre (Table III). Ulmus alata (winged elm), Quercus stellata and Carya texana were the common seedlings and saplings in both stands (Table IV). Vines were the more common members of the understory, principally Parthenocissus quinquefolia (Virginia creeper), Rhus toxicodendron (poison ivy), Smilax spp. (green briar) and Vitis spp. (wild grape). Species of Vitis had their highest importance in the eastern stand, whereas smilax was found only in the western stand (Table V).

Soils of these two stands, like those of the Woodbine stands, were much alike (Table VI). It seems probable that the slight differences in the nutrient content values of the four sandy soils are differences due to chance sampling.

The vegetation and soils of the four stands on the Woodbine and Pawpaw sands were alike in many respects. of the three black oaks, Quercus marilandica, $Q$. velutina, and Q. falcata, that occurred in these 
stands, Q. marilandica is able to grow in dryer habitats, with the other two oaks in progressively more mesic habitats. Preston (1961) described the habitats of these three species as $Q$. marilandica, dry sites; $Q$. velutina, dry to moist sites; and Q. falcata as dry to wet sites. If the relative abundance of these three species and Carya tomentosa in a stand is indicative of the degree of xeric conditions in that stand, the Pawpaw stands were slightly more xeric in nature than the Woodbine stands and the eastern stands of both a little more mesic than their western counterpart. (Table II). This might have been simply a response to the lower average annual precipitation in the western part of the area of four inches.

\section{Weno Formation Stand}

vegetation was composed mainly of Quercus stellata (post oak), Fraxinus americana (white ash), and Ulmus alata (winged elm). This stand had a basal area of $62 \mathrm{sq}$. ft. per acre which was third lowest. Its mean d.b.h. was 6.1 inches (Table III). This combination of species was similar to a stand discussed by Hutcheson (1965). The community seemed to be maintaining itself as most seedlings and saplings belonged to the three major species. Species of Cretaegus (hawthorn) were the most common shrubs and species of Smilax

(greenbriar) were the common vines.

According to soil

surveys and geological

descriptions (Olson 1965), the soil from this geological material should have been more like that of the sandy formations than my analysis indicated. Except for its coarse texture and $\mathrm{pH}$ values, this soil was certainly more closely allied to soils of the limestone and clay stands, although its nutrient values were generally lower (Table VI). The soil volumeweight was highest of any stand.

Goodland Limestone had several different types of plant communities growing on soils from this formation. In the study stand, Carya texana (black or pignut hickory) and Quercus stellata (post oak) had the highest importance percentage, but it had the highest species diversity with 17 different tree species counted in the sample. Quercus falcata (spanish oak) was the main secondary species (Table II). This stand had the highest number of trees, 277.5 per acre, but since its d.b.h. of 6.8 inches was relatively low, the basal area per acre was only $83 \mathrm{sq}$. ft. Other stands of similar composition were located in Marshall, Choctaw, and McCurtain Counties. Ulmus alata (winged elm), Fraxinus

Taylor, R.J. 
americana (white ash), and Celtis laevigata (hackberry) were reproducing extensively (Table IV). There were a number of species of shrubs, but Crataegus spp. (hawthorn) were the most abundant. Common vines were Smilax (greenbriar), Rhus toxicodendron (poison ivy), and Parthenocissus quinquefolia (Virginia creeper) (Table V) .

Although the soil had a very high sand content; soil color, $\mathrm{PH}$, the general fertility was much like that of soils from the other limestones and clays (Table VI).

\section{Bennington Limestone}

Stand vegetation was composed mainly of Celtis laevigata. (hackberry), Maclura pomifera (osage orange, bois d'arc, horse apple) and species of Ulmus (elm). Gleditsia triacanthos (honey locust) was also an associate member. In basal area per acre and soil texture this stand, like that of the Goodland limestone, was similar to those of the sandstones. Its major species were those common to the Duck Creek, Kiamichi, and bottomlands. Celtis and Maclura had the highest number of seedlings and saplings (Table IV). Symphoricarpos orbiculatus (coral berry, buckbrush) was the most common shrub, with Berchemia scandens (rattan vine) and Smilax spp. (greenbriar) the more common vines (Table V).
This soil, like that of the Goodland, had a relatively high sand-silt content. The soil of the Weno formation was the only soil containing a larger percentage of silt. The soil from the Bennington limestone had a slightly lower

nitrogen, organic carbon, and organic matter content; but it was still similar to the other limestone and clay soils (Table VI). It was interesting that the soils of the Weno Formation, Goodland and Bennington limestones were all relatively coarse textured, have poorly developed profiles, and were shallow with rock at the surface in places. Dix (1959) pointed out shallow soils often have only $A$ and D horizons. Black (1957)

stated that while sand and silt fractions might represent residual unweathered or physically weathered material, the clay was more dependent on the processes of chemical weathering .

\section{Duck Creek Limestone}

stand was composed of Ulmus crassifolia (cedar elm) and Maclura pomifera (bois d'arc). Ulmus crassifolia was often encountered growing in tight shallow, stony, clay soil in upland stands, but it was more commonly found in bottomlands. The basal area of $26 \mathrm{sq}$. ft. per acre was by far the lowest of any stand, largely because of an average d.b.h. of only 5.7 inches. Both major species were 
maintaining their importance as indicated by seedlings and saplings (Table IV). The only understory species in this stand was Cocculus carolinus (snail seed) (Table V) .

Much of the soil on this formation is under cultivation or had been cleared of woody species for meadows or pastures. The study stand had evidently developed since cultivation was abandoned about 30 years ago. This dark gray to graybrown, moderately alkaline, clay loam was relatively high in soil nutrients. It had the lowest volume-weight of the limestone and clay soils (Table VI).

Kiamichi Clay stand had only two species obtained in the sample of this stand. They were Ulmus crassifolia (cedar elm) and Maclura pomifera (bois d'arc). A few trees of Fraxinus pennsylvanica (green ash) occurred but it was not frequent enough to be included in the sampling. Basal area per acre was 62 sq. ft. and its mean d.b.h. was 5.7 inches. The three above species constituted the major portion of seedlings and saplings (Tables II and IV). Both this sand and that of the Duck Creek are variations of the same forest type. Symphoricarpos orbiculatus (coral berry, buckbrush) was the main understory species.
Similarities in the physical properties of the soil of the Kiamichi Clay and Duck Creek Limestone are to be expected, since the former is derived in a large measure from clay and lime cement of the oyster shell limestone, while the Duck Creek is formed mainly from soft chalky limestone and clay. These stands are about onehalf mile apart, thus have similar climatic conditions. The soil of the Kiamichi was a gray brown, neutral, rather tight waxy clay that had about the highest fertility of any stand (Table VI). The stands of the last two formations were also shallow with poorly developed profiles.

\section{DISCUSSION}

A total of 32 species were encountered in the samples of the 13 stands, with Cretaegus spp.

considered as one species, and 49 species of trees are listed in my field notes for Bryan County. Of the 32 species of trees, only 13 had an importance percentage of 15 or more in at least one stand. There were four other species with an importance percentage of less than 15, but more than five.

The species in this study can be placed in three categories: those that occur predominantly in the bottomlands; species that occur mainly on soils derived from sandstone; and those

Taylor, R.J. 
that occur on soils derived from limestones and clays. It is evident that some overlap occurs as the Goodland limestone stand species seem more closely related to the species on the sandstones, while those on the Bennington limestone and clays seemed more closely allied to those of the bottomlands. There were 14 species that had their highest importance percentages or occurred only in stands on predominantly coarse textured soils. Soils predominantly of sand and silt are considered as coarse textured soils (Lyon and Buckman 1943, Black 1957, and Russell 1957). Of the 14 species, there were nine with an importance percentage of at least five per cent in one or more stands. There were 13 species that had their highest importance percentage or occurred only in stands that grew in soils which were mainly fine textured. Lyon and Buckman (1943) stated that a soil with at least 30 per cent clay was considered a clay soil. This would include soil of the three bottomlands, the Duck Creek limestone, and Kiamichi Clay. of these 13 species, seven had an importance percentaage of 5 per cent or more. The stands with the largest average tree size and highest basal area were the bottomlands, whereas the highest number of trees per acre occurred on the Goodland Limestone and the Kiamichi clay. The most open stand was the Clear Boggy Creek stand. The average basal area per acre for all stands was 78.6 square feet $(23.96$ $\mathrm{m})$ per acre. The average for the three bottomland stands was $99.5(30.32 \mathrm{~m})$, that of the stands on sandstone derived soils was 77.2 (23.53 $\mathrm{m})$, and the average of stands on limestone and clay soils was $65.1(19.84 \mathrm{~m})$. The bottomland stands were by far the most productive. The basal area per acre of the Goodland and Bennington limestone stands was more like that of sandy soil stands rather than the clay soil stands. If total nitrogen, total phosphorus and organic carbon were used as an index of fertility, stands growing on soils derived from limestone and clay were the least productive, but grew on the most fertile soil. The average basal area per acre for 13 stands of this study was essentially the same (78.74 sq. ft.) as that found by Taylor (1965) for an 80 acre stand growing on the Antlers Sand. His study was conducted approximately eight miles west of the Antlers sand stand of this study. Rice and Penfound (1959) found an average basal area per acre of only $55.2 \mathrm{sq}$. ft. for their three Bryan county sands. Two of their stands were on soils derived from the Woodbine sand, while the third was underlain by the 
Pawpaw sand. They reported average basal area per acre as follows: Quercus stellata (post oak) 23.0; $Q$. marilandica (blackjack oak) 77.7; Q. velutina (black oak) 10.2; Carya texana (black or pignut hickory) 9.4. The average basal areas per acre of those species in the four stands of this study on the same formations were 26.7, $7.2,12.8$, and 19.3 square feet respectively. The eastern stands of the Woodbine and Pawpaw formations seemed to be slightly more mesic than the western ones on the same formations. The Woodbine stands appeared bo be a little more mesic than the Pawpaw stands.

Using the same criteria for seedlings and saplings as that used for tree species, there were a total of 34 species encountered in all stands. Four of the 34 were not encountered as trees, and two species tabulated as trees were not found as seedlings and saplings. Only 14 species had an importance percentage of 15 or more in at least one stand. Of this number Morus rubra (red mulberry) and Salix nigra

(black willow) had lower percentages as trees. Sapindus drummondii (soapberry) was not tallied as a tree for any stand. Seedlings and saplings of Platanus occidentalis (sycamore) and Quercus macrocarpa (bur oak) were not tallied although they

occurred as trees.

There were 14 species of shrubs and 10 vines tallied for all stands, whereas my field notes list 34 shrubs and 18 vines for the Bryan county area. In general vines were much more common than shrubs in the study stands. Only two species of shrubs were found in the Kiamichi clay and none in the Duck Creek Limestone stand. Both had only one species of vine, Cocculus carolinus (snail seed).

The types of forest communities of interest encountered in this study were the post oak-white ashwinged elm and the hackberrycedar elm-green ash. The first has only recently been described from the Arbuckle mountain area by Hutcheson (1965). The latter does not seem to have been reported for Oklahoma.

When the soil factors of the stands were analyzed, it was found that the soils derived from 1) sandstone 2) limestone and clay, and 3) bottomlands differed considerably. Soils of the latter two were much alike in a number of factors and were sharply distinct from the sandstones except for $\mathrm{pH}$ and soil volume weight. The Goodland limestone and Bennington limestone had soil textures that were more like those of the sandstones than the other soils. These two, with the soil from the Weno

Taylor, R.J. 
formation, might have been placed in a separate category. The soil reaction $(\mathrm{pH})$ ranged from slightly acid to moderately alkaline (6.2-8.0). The $\mathrm{pH}$ of most stands were within or close to the neutral range, 6.67.2, for soils (Gray and Galloway 1959), and probably was not sufficiently high or low to be critical in any of the stands. Gray and Galloway (1959) stated that a $\mathrm{pH}$ range of 6.1 to 7.3 is optimum for the growth of most organisms. Although the sandstone soils were much lower in fertility, all but the Pawpaw east and Weno formation soils had their colloidal complexes essentially base saturated (Eyre 1963). Black (1957) stated that soil nitrogen increased as the soil texture became finer. If the clay content is used as an index of soil texture, the Goodland limestone with only 24 per cent clay provided an exception. It had the next to highest amount of nitrogen, 5,450 lbs. per acre, whereas the Clear Boggy Creek soil with 67 per cent clay had only 3,666 lbs of nitrogen per acre. When the colloidal portion of organic matter, which may have had an exchange capacity twice that of some clays (Thompson 1952), and the clay portion were considered together, the texture and nitrogen relationship correlated well. The same correlation existed with soil phosphorus. There also seemed to be some correlation between these factors and soil volume weight.

There were nine species of trees that occurred mainly on coarse textured soils, and had their highest importance percentages on sandy soils which were lowest in total nitrogen and phosphorus. They were Quercus stellata (post oak), Q. velutina (black oak, ) Q. marilandica (blackjack oak), Q. falcata (spanish oak), Ulmus alata (winged elm), Carya texana (black or pignut hickory), $C$. tomentosa (now C. alba, mockernut hickory), Cercis canadensis (redbud), and $U$. americana (american elm). Fraxinus americana (white ash), Quercus shumardii (Shumard's red oak), and $Q$. muehlenbergia (Chinquepin oak) may also belong in this group although they occurred on soils with relatively high soil fertility as did $Q$. stellata, Carya texana, and U. alata.

Four species, Celtis laevigata (hackberry), Fraxinus pennsylvanica (green ash), Maclura pomifera (bois d'arc), and Ulmus crassifolia (cedar elm) had their highest importance percentages on fine-textured soils, both in bottomlands and uplands. All but U. crassifolia had seedlings on most of the coarse textures soils. A number of the remaining species appeared to be restricted to bottomlands with an occasional occurrence 
in upland stands. Anderson (1954) suggested that a number of species of trees, such as elms, sycamore, and honey locust, which are usually found in bottomlands, occasionally occurred in uplands where some kind of disturbance laid bare the topsoil. Bottomlands that flood seasonally were areas continually disturbed by inundation and deposition of silt and clay. The shallow soils of the Bennington limestone, Duck Creek limestone, and Kiamichi clay are probably very susceptible to disturbance. A number of pioneer species, which often become weedy, are known to have a wide ecological amplitude (Harlan and deWet 1965), Foote and Jackobs (1966) found Cassia fasciculata (partridge pea), for example, on soils with a wide range of ecological conditions. This may also be the case of such tree species as Celtis laevigata, Diospyros virginiana (persimmon), Fraxinus pennsylvanica, Gleditsia triacanthos, Maclura pomifera, Ulmus alata, and $U$. crassifolia. The committee on forest types (1931) listed some of these species in both bottomland and upland communities, some of which occurred on dry limestone hills. If this hypothesis is correct, it would help to explain why some of the tree species could be important components in both bottomland and dry uplands. Ulmus crassifolia, near the northern edge of its range, was found as a tree mainly on soils containing more than 30 per cent clay, whereas $U$. alata occurred as a tree on soils having less clay. Although both species occurred in many kinds of habitats, both bottomland and upland, U. crassifolia apparently prefer red finetextured soils, U. alata coarse-textured soils.

In general the distribution of seedlings and saplings corresponded to the soils on which trees of that species also occurred. The number of seedlings and saplings was low in the two Red River stands, the Duck Creek limestone, and Kiamichi clay. Shrub and vine species in the study did not seem to be restricted to any soil type. This agreed with the findings of Hutcheson (1965) The one soil factor which seemed to be most influential in the distribution of species of trees as found in this study was soil texture. Soil texture, however, either directly or indirectly affected most other soil factors, including rate of water infiltration, available water, soil aeration, and soil nutrient content (Black 1957). Soil texture, as well as a number of other soil properties, were known to be related to the geological material from which it was

Taylor, R.J. 
formed (Gray et al. 1959). Usually coarse-textured soils developed from sandstone, and other sandy material, whereas fine-textured soils developed from such materials as clays, marls, and soft limestone. Quarterman and Keever (1962) found sandy surfaced soils developed above a number of different kinds of materials including limestone. They found no correlation between soil fertility and types of forest stands. In this study coarse-textured soils were found on the sandstone formations, Weno formation, Bennington limestone, and Goodland limestone; whereas fine-textured soils were found on the alluvium, Duck Creek limestone and Kiamichi clay.

\section{SUMMARY}

In this study the vegetation of 13 forest communities were analyzed. The soil in which they grew was also studied and the communities correlated with soil and geological material. They were all located in the Bryan County portion of the Oklahoma Gulf Coastal Plain. These forest stands were established on the following geological formations: Clear Boggy Creek alluvium, Red River alluvium, Antlers sand, Pawpaw sand, Woodbine sand, Weno formation, Goodland limestone, Bennington limestone, Duck Creek limestone and Kiamichi clay.
Extensive reconnaissance resulted in the selection of 13 forest stands on the above formations. Sampling was accomplished by the pointcentered quarter method, with the number of plants, basal area of trees, and importance percentage as the most useful vegetation parameters. Soil

factors studied included soil texture, soil reaction, amounts of organic carbon, total nitrogen, and total phosphorus, and the degree of soil compaction.

of the 13 stands, three

grew in bottomlands, six grew on sandy substrates, and four occurred on limestone or clay derived soils. In addition these stands allowed comparison of forest communities growing on the same formations 40 miles apart, and two different stages of succession. In the bottomland communities, the species with the highest importance percentages were celtis laevigata (hackberry), Fraxinus pennsylvanica (green ash), Populus deltoides (cottonwood), Ulmus crassifolia (cedar elm), and U. rubra (slippery elm). The most important species growing in stands on coarse textured soils, including the Goodland limestone stand, were Carya texana (black or pignut hickory), Quercus falcata (Spanish oak), $Q$. marilandica (black jack oak), $Q$. stellata (post oak), $Q$. velutina (black oak), and Ulmus alata (winged elm). 
The dominant species on the Kiamichi clay, Bennington and Duck Creek limestones were Celtis laevigata, Maclura pomifera (bois d'arc), and Ulmus crassifolia. On the basis of basal area, the bottomlands were the most productive, sandy soils intermediate, and upland fine-textured soils least productive, although with the highest nutrient content.

In comparing the two stands representing early and late stages of succession, it was found that physical properties of their soils were very similar, but that the soil of the mature stand was more fertile and more productive. The soil properties of the Pawpaw and Woodbine sand formations were similar both in physical and chemical properties, although they were 40 miles apart. The eastern stands were slightly more mesic.

As a general rule, seedlings and saplings of the overstory species were abundant in all stands except the cottonwood stand on the Red River alluvium. Shrubs and vines were common, but not abundant in all stands. Vines were more numerous in most communities than shrubs. Based on the results of this study, the most important soil factor influencing the distribution of trees and forest communities was soil texture. No correlation between soil type and either shrubs or vines was observed.

\section{ACKNOWLEDGMENTS}

The writer wishes to express his sincere thanks to Dr. Wm. T. Penfound under whose direction this study was done. Thanks are also extended to Dr. Carl D. Riggs, Director, University of Oklahoma Biological Station for the use of equipment and to the National Science Foundation for financial assistance during the summer of 1963, when the soil analysis were done. My appreciation is extended to the various property owners of the study stands for their cooperation, and information concerning history of the study areas.

Last, but not least, I wish to thank my wife, Connie, for help in collecting part of the data and in preparation of this manuscript.

\section{LITERATURE CITED}

Anderson, Edgar. 1954. Plants, Man and Life. Andrew Melrose Limited, London .

Beadle, N. C. W. 1966. Soil phosphate and its role in molding segments of the Australian flora and vegetation, with special references to xeromorphy and sclerophylly. Ecology 47:922-1007.

Beals, E. W. and J. B. Cope. 1964. Vegetation and soils

Taylor, R.J. 
in an eastern Indiana

woods. Ecology 45:777-792.

Black, C. A. 1957. Soil- Plant Relationships. John Wiley \& Sons, Inc., New York.

Blair, W. F., and T. H. Hubbell. 1938. The biotic districts of Oklahoma. Amer. Mid. Nat. 20:425-454.

Blan, P. E. 1961. Petrology of the Goodland Limestone of southeastern Oklahoma. Unpub. M. S. Thesis, University of Oklahoma, Norman.

Bouyoucos, G. J. 1936. Directions for making mechanical analyses of soils by the hydrometer method. Soil Sci. 42:225230 .

Braun, E. Lucy. 1964. Deciduous Forest of Eastern North America. Hafner Publ. Col, New York.

Bruner, W. E. 1931. The vegetation of Oklahoma. Ecol. Monogr. 1:99-188.

Buck, Paul. 1964.

Relationships of the woody vegetation of the Wichita Mountains Wildlife Refuge to geological formations and soil types. Ecology $45: 331-344$.

Bullard, F. M. 1925. Geology of Love County, Oklahoma. Okla. Geol. Survey, Bull. 33:77 p. - 1926. Geology of

Marshall County, Oklahoma. Okla. Geol. Survey, Bull. 39:101 p.

Cottam, G. and J. T. Curtis. 1956. The use of distance measures in phytosociological sampling. Ecology 37:451-457.

Cottom, G., and J. T. Curtis, and B. W. Hale. 1953. Some sampling characteristics of a population of randomly dispersed individuals. Ecology 34:741-757.

Crockett, J. J. 1964. Influence of soils and parent material on grasslands of the Wichita Mountains Wildlife Refuge, Oklahoma. Ecology 45:326335 .

Curtis, N. E., Jr. 1960. Lignite in the Red Branch Member, Woodbine Formation, Oklahoma. Okla. Geol. Survey, Okla. Geol. Notes $20: 240-244$.

Cuyler, R. H. 1931. Vegetation as an indicator of geologic formations. Bull. Am. Assoc. of Petr. Geologists $15: 67-75$.

Davis, L. V. 1960. Geology and ground-water resources of south McCurtain County, Oklahoma. Okla. Geol. Surv. Bull. 86:108 p.

Dix, R. L. 1959. The influence of grazing on the thin-soil prairies of Wisconsin. Ecology 40: 36-49.

Duck. L. G., and J. G. Fletcher. 1943. A game type map of Oklahoma. Oklahoma Game and Fish Dept., Oklahoma City.

Duck L. G., and J. G. Fletcher. 1945. Survey of the Game and Furbearing animals of Oklahoma. Oklahoma Game and Fish Dept. Oklahoma City.

Dwyer, D. D., and P. W. Santelmann. 1964. A comparison of post oakblackjack oak communities on two major soil types in north central Oklahoma. Okla. Exp. Sta. Bull. B$626,15 \mathrm{p}$. 
Eyre, S. R. 1963. Vegetation and Soils. Aldine Publ. Col, Chicago, Illinois

Fenneman, N. M. 1938. Physiography of eastern United States. McGraw-Hill Book Col, New York.

Foote, L. E., and J. A. Jackobs. 1966. Soil factors and the occurrence of partridge pea (Cassia fasciculata Michx.) in Illinois. Ecology $47: 968-975$.

Forgotson, J. M. 1957. Stratigraphy of Comanchean Cretaceous Trinity group. Amer. Assoc. Pet. Geologists Bull. 41:23282363.

Gibbs, H. D. 1950. A field study of the Goodland Limestone and the Washita Group in southeastern Choctaw County, Oklahoma. Univ. Oklahoma unpublished Masters Thesis. Norman, Okla.

Gray, Fenton and H. M. Galloway. 1959. Soils of Oklahoma. Oklahoma State Univ. Ex.. Stat., Misc. Publ MP-56. Stillwater, Okla.

Hall, M. T. and C. J. Carr. 1964. Differential selection in Juniper populations from the Baum limestone and Trinity sand of Southern Oklahoma. Butler Univ. Botan. Studies $14 ; 21-40$.

Harlan, J. R., and J. M. J. deWet. 1965. Some thoughts about weeds. Economic Botany 19:16-24. Hedlund, R. W. 1962. Palynology of the Red Branch Member of the Woodbine Formation

(Upper Cretaceous) in Bryan County, Oklahoma. Unpubl. Ph.D. Dissertation. Univ. of Oklahoma, Norman.
Heilborn, G. 1949. Stratigraphy of the Woodbine Formation, McCurtain County, Oklahoma. Unpubl. Masters Thesis, Univ. of Oklahoma, Norman. Hutcheson, H. S. 1965. Vegetation in relation to slope exposure and Geology in the Arbuckle Mountains. Unpubl. Ph.D. Dissertation. Univ. of Oklahoma, Norman.

Kuchler, A. W. 1964. Potential Natural Vegetation of the Conterminous United States. Amer. Geogr. Soc. Spec. Publ. No. 36. $116 \mathrm{p}$. \& map.

Little, E. L. 1938. The vegetation of Muskogee County, Oklahoma. Amer. Mid. Nat. 19:559-572

Lyon, T. L., and H. C. Buckman. 1943. The Nature and Properties of Soils. Macmillan Co., New York. $499 \mathrm{p}$.

Melton, F. A. 1954. Natural mounds of northeastern Texas, southern Arkansas, and northern Louisiana. Okla. Geol. Survey, "Hopper" 14: No. 7.

Miser, H. D. 1927. Lower Cretaceous rocks of southeastern Oklahoma and southwestern Arkansas. Amer. Assoc. Petrol. Geologists. No. 11. - 1954. Geologic Map of Oklahoma. Okla. Geol. Surv., Norman, Oklahoma.

Mooney, H. A. 1966. Influence of soil type on the distribution of two closely related species of Erigeron. Ecology 47:950958.

Morisita, Masaaki. 1954. Estimation of population density by spacing method. Memoirs of the Fac. of

Taylor, R.J. 
Sci., Kyushu Univ., Series E (Biology) 1:187-196.

Noggle, G. R. and F. L. Wynd. 1941. The determination of selected chemical characteristics of soil which affect the growth and composition of plants. Plant Physiol. 16:39-60.

Olson, L. J. 1965. Geology of Eastern Bryan County, Oklahoma. Unpublished Masters Thesis, Univ. of Oklahoma, Norman, Okla.

Piper, C. S. 1944. Soil and plant analysis.

Interscience Publishers, Inc., New York. 368 p.

Porter, C. L. 1966. An analysis of variation between upland and lowland switchgrass, Panicum virgatum L., in central Oklahoma. Ecology 47:980992.

Preston, R. J. 1961. North American Trees. Iowa

State University Press, Ames, Iowa. 395 p.

Quarterman, Elsie, and Catherine Keever. 1962. Southern Mixed Hardwood forest: climax in the southeastern coastal plain: U. S. A. Ecological Monographs $32: 167-185$.

Redman, R. H. 1964. PostMississippian Geology of Love County, Unpubl. Masters Thesis. Univ. of Oklahoma, Norman, Okla.

Rice, E. L., and Wm. T. Penfound. 1959. The upland forest of Oklahoma. Ecology 40: 593-608.

, Wm. T. Penfound, and L. M. Rohrbaugh. 1960. Seed distribution and mineral nutrition in succession in abandoned fields in central Oklahoma. Ecology 41: 224-228.

Shed, J. S. and Wm. T. Penfound. 1964. Distribution of legumes as correlated with surface geology and plant succession. Proc. Okla. Acad. Sci. 44:2-6.

Shelton, W. R. and R. J. Harper. 1941. A rapid method of the determination of total phosphorus in soil and in plant material. Iowa state Coll. Sci. 15:403-413.

Skolnick, N. 1949. Lithology and Stratigraphy of the Tokio Formation of McCurtain County, Oklahoma. Unpubl. Masters Thesis, Univ. of Oklahoma, Norman, Okla.

Society of American Foresters. 1931. Forest Cover Types of the eastern United States. Soc. Amer. For., Washington, D.C. 39 pp. Stephenson, L. W. 1919. A contribution to the geology of northeastern Texas and southern Oklahoma. U.S. Geol. Survey, Prof. Paper 120-N:129-163.

Taff, J. A. 1902. Description of the Atoka quadrange. U.S. Geol. Survey, Geol. Atlass, Folio 79. 1903. Description of the Tishomingo quadrangle. U.S. Geol. Survey, Geol. Atlas, Folio 98.

Taylor, John. 1965. Shortleaf Pine (Pinus echinata) in Bryan County, Oklahoma. Southwestern Naturalist $10: 42-47$.

Taylor, R. John, and Wm. T. Penfound. 1961. The Grassland communities on the Baum Limestone in Johnston County, Oklahoma. Southw. Natur. 6:98-99. 
Taylor, R. John and Constance Taylor. 1965. Additions to the Vascular flora of Oklahoma. Rhodora 67:191193.

Thompson, L. M. 1952. Soils and Soil Fertility. McGraw-Hill Book Co., Inc., New York.

Thornthwaite, C. W. 1948. An approach toward a rational classification of climate. Geogr. Rev. 38:55-94.

U.S. Dept. of Agric. 1941. Climate and Man. Yearbook of Agriculture. U.S. Govt. Print. Office, Washington, D.C. 1248 p.

U.S. Dept. of Agric. 1957. Soil. Yearbook of Agriculture, U.S. Govt. Print. Office, Washington, D.C. Pages 31-37.

U.S. Dept of Agric. 1965. Climatic Summary of the United States. Supplement for 1951-1960. Oklahoma.
Climatography of the United States No. 86-30. 88 p. Vanderpool, N. D. 1928. A preliminary study of the Trinity Group in southwestern Arkansas, southeastern Oklahoma, and northern Texas. Amer. Assoc. Patr. Geologists Bull. 12:1069-1094.

Waterfall U. T. 1966. Keys to the flora of Oklahoma. Dept. of Botany and the Research Foundation, Okla. State Univ., Stillwater, Okla.

Wright, R. D., and H. A. Mconey. 1965. Substrata-oriented distribution of Bristlecone Pine in the White Mountains of California. Amer. Mid. Nat. 73:257-284.

Zinke, P. J. 1962. The pattern of individual forest trees on soil properties. Ecology $43: 130-133$.

Taylor, R.J. 